\title{
Scalable Modular Self-reconfigurable Robots Using External Actuation
}

\author{
Paul J. White and Mark Yim
}

\begin{abstract}
This paper presents a method for scaling down the size and scaling up the number of modules of selfreconfigurable systems by focusing on the actuation mechanism. Rather than developing smaller actuators, the main actuator is removed entirely. Energy instead comes from the environment to provide motion in prescribed synchronous ways. Prescribed synchronous motions allow much faster assembly times than random Brownian motion which has been used before. An instantiation of this idea is presented using a motion platform to induce motions based on the inertial properties of the modules and the timed actuation of small latching mechanisms.
\end{abstract}

\section{INTRODUCTION}

One of the goals for modular self-reconfigurable (MSR) robot research is to increase the numbers of modules and decrease their size. MSR's robot systems are made up of many repeated modules that can rearrange their shape [1]. Many of the lattice based MSR systems can approximate arbitrary shapes [2]-[4].

In telepario [5] the robots modules act to mimic the $3 \mathrm{D}$ shape of another 3D scene, much like a TV screen mimics a 2D image. The precision with which these modules can approximate shapes is a function of the size of the individual modules, thus the desire to reduce size. In addition, smaller modules may enable functionality that larger modules cannot. For example, in robotic tasks, smaller modules may be able to squeeze under a door, or inside the lock on a door.

One major design obstacle to shrinking down modules is the size taken up by actuation. In many cases, the actuators and associated transmissions are made as small as possible and are still more than $50 \%$ of the volume and weight of a module[1][9]. Actuation in a self-reconfigurable module nominally consists of a main actuator, that moves the module (or neighboring modules), and a latching/unlatching actuator. Usually, the main actuator takes the largest amount of volume since it typically has largest demands of force or torque.

There has been speculation that micron or millimeter sized modules could be constructed using electrostatics for actuation, however, none has yet been created. Using MEMS technologies, a planar version might be conceivable however, power distribution, release of modules and onboard computation are just a few of the technical difficulties in implementing such a device. Even if these systemic issues are achieved, it's not clear that achievable electrostatic actuation would overcome friction/stiction forces, nor scale to 3-D.

Instead of developing new actuation technologies this paper proposes to remove the main actuator from the module and use forces from the environment to provide the main propulsion for modules. Currently other existing work in

Manuscript received April 8, 2007

P.J. White and Mark Yim are with the Mechanical Engineering and Applied Mechanics Department at the University of Pennsylvania (e-mail: whitepj,yim@seas.upenn.edu). this area use similar ideas of external motive force, but use random "brownian" motion [7],[8]. This suffers from very long, indeterminate assembly times. For applications like telepario this type of assembly will not fit the realtime requirements. We propose to use an explicit controlled directionable external force that will provide fast assembly times for an arbitrary number of modules while minimizing internal module actuation.

This paper develops components of a system that will self-reconfigure utilizing forces provided externally with the general shape formation problem as the focus application.

\section{HARDWARE AND DESIGN}

\section{A. Mechanical}

The concept of external actuation is simply to drive the environment of a system of modules with a specific energy pattern that causes the modules to reach a given goal state. Where typical modules in modular robotic systems can both actuate their bonding mechanisms and reconfigure to a different location in the structure, the modules in an externally actuated system need only a bonding mechanism. In this way, the modules determine the course of reconfiguration while the environment provides the necessary energy to do so.

In order to explore the design issues for externally actuated systems, this paper presents a simple physical implementation. The actuated environment includes a plane mounted to a linear stage that provides linear acceleration of the plane. One module is fixed to the center of the plane and other modules form a lattice structure around that module. Two modules (shown in Figure 2) bond to one another with two pairs of magnets at two edges of the cube. A module can reconfigure about one of its two magnetic bonds to its neighbor provided it does not collide with another module in the process. They initiate reconfiguration by actuating a mechanism to break the bond of a pair of magnets at one edge. This initiation is synchronized with the acceleration of the table. The inertia of the modules provide the necessary mechanism to cause the module to move, while the one remaining magnetic bond constrains the motion to rotate about the edge to a new position in the structure. A typical reconfiguration sequence is shown in Figure 4.

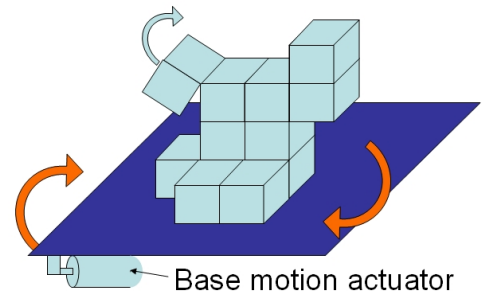

Fig. 1: Concept illustration: a 3D modular self-reconfigurable robotic system driven by external actuation. 
A critical design aspect that is common to all MSR robots is a switchable bonding mechanism. It must provide enough force so modules will remain attached to the structure under the range of operating conditions. However, this mechanism must also be able to un-bond so that the module can reconfigure. Several methods have been employed [9][11] ranging from electro-magnetics to mechanical latches. In the external actuation method, the modules reconfigure dynamically, so the restraint force of the inter-module bond must be sufficiently high in proportion to the inertia of the module. At the same time it must leave one rotation degree of freedom (DOF) free for the modules to reconfigure.

Given these constraints, the following materials were chosen to construct a switchable bonding mechanism: (1) neodymium magnets (bonding force), (2) Shape Memory Alloy (SMA) wires (force to break bond), and (3) an ABS plastic structure (compliant mechanism). The bonding method must be able to provide a enough holding force such that the inertial forces during reconfiguration don't break the bond while allowing rotation about one edge. Cylindrical magnets were chosen both for their high force to weight ratio and their curved geometry which is amenable to the dynamic rotation.

The structure of the module consists of a pair of planar ABS frames that are joined together to form and ' $X$ ' shape. As seen in Figure 2, each leg of the module has a pair of compliant arms with magnets at the tip used to bond one module to another. In addition, each arm is attached to an SMA wire for actuation that is linked to a fixed part of the frame. The design of the compliant arm is essentially a beam-in-bending problem constrained by two SMA wire limitations: the maximum force that it can provide is $20 \mathrm{~N}$ and to prevent overstress, the maximum strain in contraction must be limited to less than $4 \%$. The goal is to determine an appropriate geometry (and thus spring constant) for the arm such that it retracts sufficiently to break the magnetic bond yet does not allow the material to exceed the yield strength of ABS (roughly $40 \mathrm{MPa}$ ). Several finite element (FEM) analysis iterations yielded a design that met the design constraints. The FEM for the final design is shown in Figure 3.

Another design aspect deals with minimizing the friction between the module and the plane. When a module reconfigures, it slides on the plane as it rotates about another module. The friction from this sliding must be overcome by the energy input by the external actuation. Each module has four rounded acetal 'feet' at the end of each of its legs. Thus, the material and minimal points of contact help to reduce the friction. In non-planar systems, modules may rotate without contact on a surface, so friction only occurs at the rotating joint and from the air.

One design goal is simplicity, with idea that lower mechanical complexity eases module size reduction. The structure is almost entirely made up of planar elements simplifying and easing manufacturability. In addition, the module is mostly solid state in that the only actuation is the deflection of the compliant bonding mechanism. The SMA wires and neodymium magnets provide a reliable, low volume, light

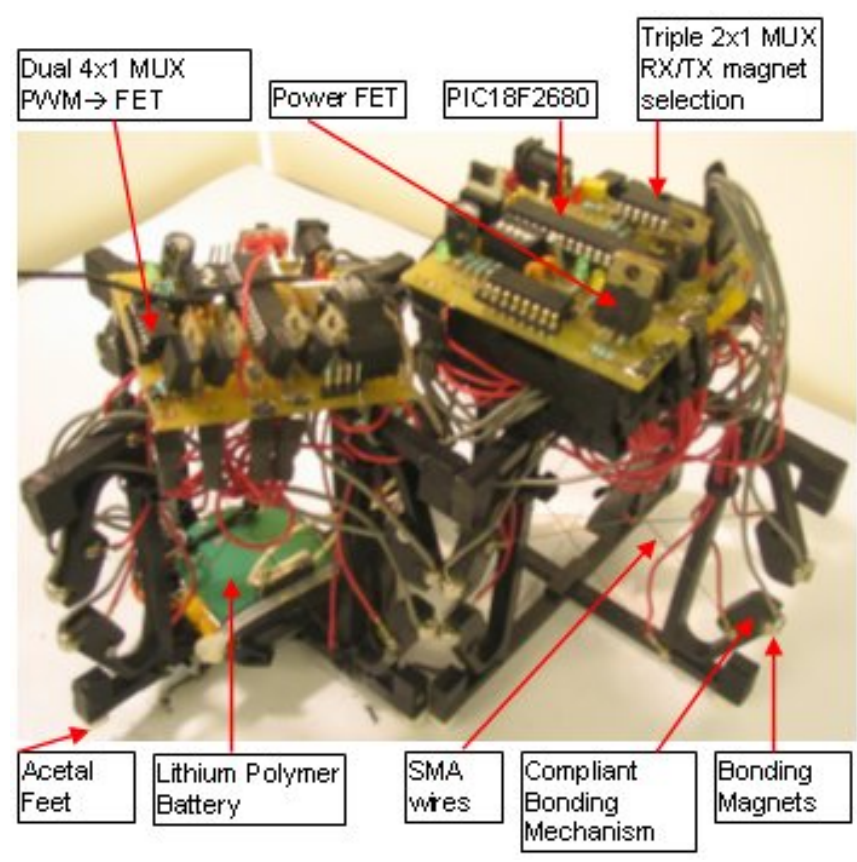

Fig. 2: Pair of externally actuated MSR robots.

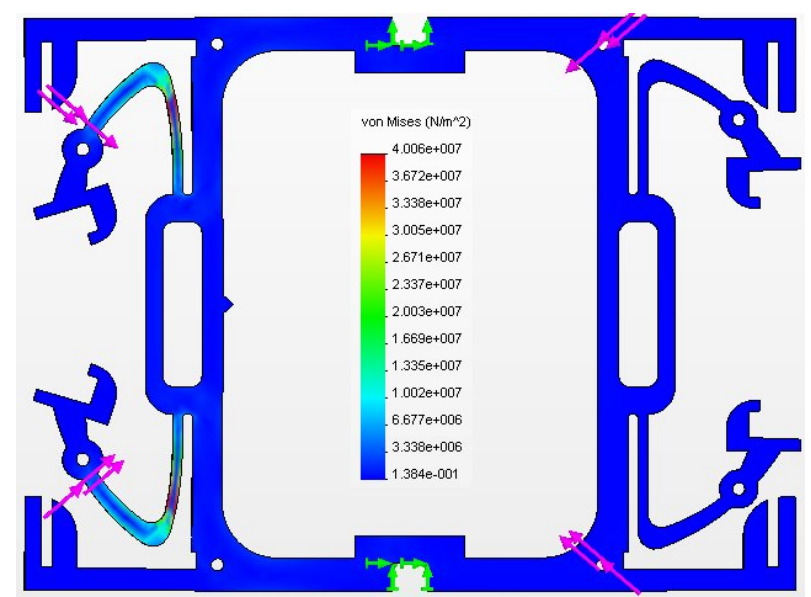

Fig. 3: Finite element analysis plot of displacement caused by SMA wire actuation force.

weight switchable bonding mechanism. The modules can be fully constructed in roughly 8 to 10 man hours at a cost of roughly $\$ 50$ in parts as shown in table I.

\section{B. Electrical}

The modules have an 18F2680 PIC microprocessor that runs the high level reconfiguration algorithm with two main functions: inter-module serial communication and control of SMA wire actuation. Modules communicate via electrical connectivity at the nickel coated magnets bonds. Of the eight magnets on a module, the four lower magnets provide a common ground for all modules while the upper four magnets alternate between transmit and receive communication. The arrangement of the magnets on a module is such that each leg of the module is the opposite polarity of the adjacent leg as shown in Figure 2. In this way the north/south magnetic parity pairs well with the transmit/receive parity required for serial communication. 
TABLE I: Cost per module at limited quantity unit cost rough

\begin{tabular}{|c|c|}
\hline item & cost in USD \\
\hline SMA & $\$ 8.00$ \\
\hline ABS plastic & $\$ 2.00$ \\
\hline Printed circuit board & $\$ 10.00$ \\
\hline CPU (pic18F2680) & $\$ 8.00$ \\
\hline Drivers & $\$ 8.00$ \\
\hline Other electronics & $\$ 8.00$ \\
\hline total & $\$ 44.00$ \\
\hline
\end{tabular}

The SMA wires are controlled via a pulse width modulated (PWM) signal that drives the gait of a power FET. The use of a PWM signal allows the amount of current passed to the SMA wires (and thus the amount of contraction) to be tuned precisely. The PWM signal from the microprocessor is routed to the gait of the FET via a $4 \times 1$ multiplexer that allows the microprocessor to select the SMA wire to contract.

One of the significant contributions of this work is that it offers a method for self-reconfiguration that greatly reduces the energy capacity requirement for each module. Often the bulk of the energy used in shape-changing systems goes to the motion of the modules which now is supplied offboard. In addition, since the weight of batteries and motors isn't multiplied by every module, the total energy used for reconfiguration is also reduced. The majority of a module's energy is used to actuate the switchable bonding mechanism. The current modules use $14 \mathrm{~W}$ to actuate the compliant arm and break the magnetic bond. The phase transition for SMA wires is relatively slow; it takes 3 seconds to contract the wire $4 \mathrm{~mm}$. However, careful design of the module and the external actuation system (discussed below) can ensure quick (less than a second) and reliable reconfigurations. Thus the nominal amount module energy required to a perform a reconfiguration is 42J. This concept is of particular importance when developing modules at scales orders of magnitude smaller where energy density becomes a critical design limitation.

The modules are powered by $7.4 \mathrm{~V}$ lithium-polymer batteries. Because the modules require a relatively large amount of power for a only short period of time at the beginning of each reconfiguration, the $730 \mathrm{mWh}$ capacity of the batteries is sufficient to allow for hours of experimentation.

\section{Communication}

The communication architecture is based on strictly local information transfer. It is not limited by the fixed bandwidth and address space of a global bus system. The communication protocol is a crucial element to the coordination of multiple neighboring modules. The modules use standard asynchronous serial communication to determine an understanding of their local state.

For the initial prototype testing with just two modules, one module is designated as a master and the other as the slave. A standard master/slave protocol is employed to handle the communication between modules in a reliable manner. Because the module is limited to communicating out of one side at a time, it must cycle through a communication sequence that attempts to communicate at each of the four sides successively until it finds another module and performs a handshake. The period for the master module is several

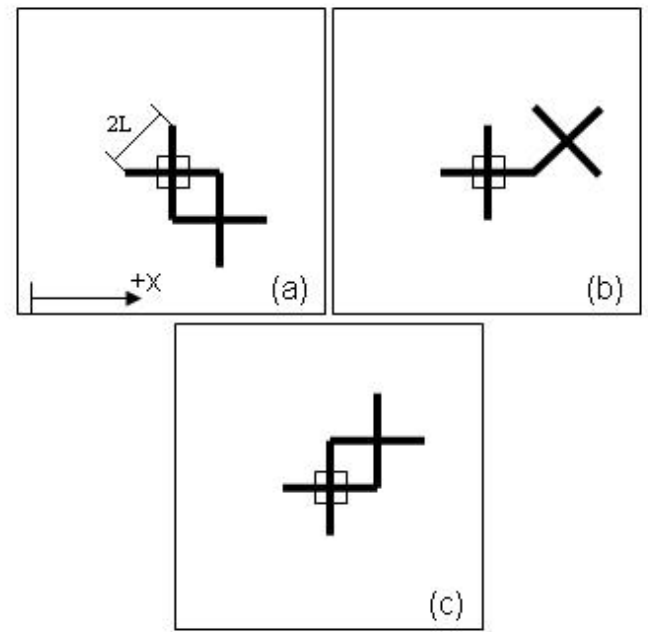

Fig. 4: Reconfiguration sequence. Table moves +/- x. Module with box is fixed. A module starts at (a) breaks one bond and rotates about other as in (b) and ends up in (c).

times greater than the period of the slave. Thus, each time the master module attempts to communicate out of a given side the slave module will cycle several times through its communication sequence. Eventually the master module and slave module synchronize and perform a handshake. This method could be extrapolated to the case with many more modules using TDMA or token passing for example and could also be extrapolated to multi-master or masterless communication methods.

\section{Design Metrics}

The development of the proof of concept brings to light many critical design issues. The key design challenge is to develop a module and an external actuation system that can reconfigure reliably. In this case, reliability is a measure of how consistently a given acceleration profile can cause a successful reconfiguration. Two critical design metrics are considered: the bonding force to inertia ratio and the precision of the external actuation system.

In order to prevent a module from detaching from the structure during reconfiguration, the bond must be sufficiently stronger than the inertial forces that might cause the bond to break. Thus, one critical design metric is the ratio of the bonding force to maximum inertial forces that are caused during reconfiguration.

The precision of the external actuation energy profile is another critical design issue. It is necessary to design a module that can reliably perform a given reconfiguration given a wide range of acceleration profiles.

The lower bound on the relative magnitude of the energy profile is dictated by the inertia and friction between the module and the environment. Minimizing these design parameters reduces the amount of energy required to cause reconfiguration. For modules that rotate in $2 \mathrm{D}$ on a plane, a metric for optimal design parameters can be derived from the expression for angular acceleration of the module given 


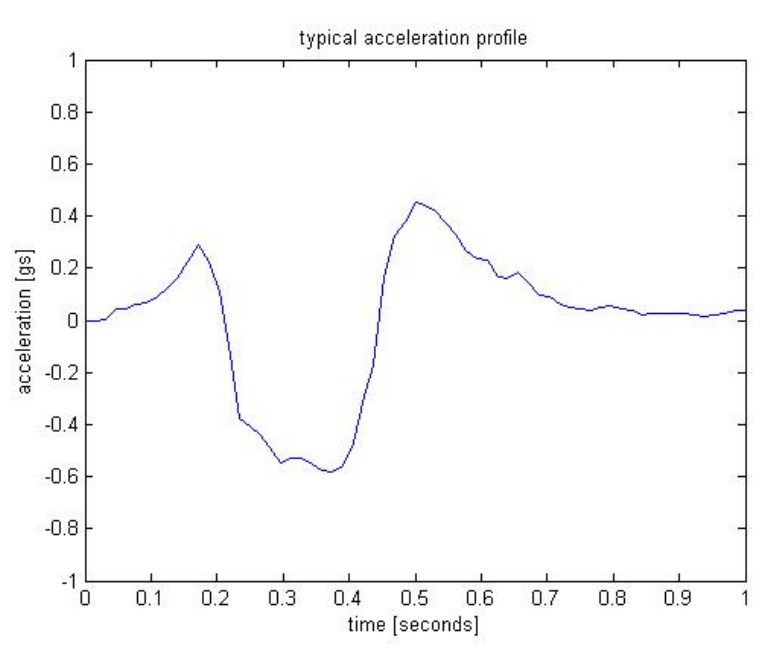

Fig. 5: Typical acceleration for reconfiguration in Figure 4

as

$$
\ddot{\theta}=\frac{3}{8 L}\left(a_{x}(\sin (\theta)-\cos (\theta))-\mu g \operatorname{sgn}(\dot{\theta})\left(1+\frac{\sqrt{2}}{2}\right)\right)
$$

where $L$ is defined in Figure 4 and $a_{x}$ is the acceleration profile in the $x$ direction. In order to maximize the angular acceleration of a module for a given $a_{x}$, the friction and the length of a side $(2 L)$ should be minimized. As the scale of the module decreases the torque due to friction will be primarily a function of area and scale as the square of the length of the modules side.

On the other extreme, the maximum energy limit is coupled to the mass, inertia and bonding force as previously discussed. Increasing the bonding force is directly correlated to the maximum energy limit. The upper limit for the maximum acceleration $a_{x}$ can be found by looking at the components of the force of constraint given by

$$
\begin{aligned}
F_{c x}= & (5 / 8 \cos (\theta)+3 / 8 \sin (\theta)) m a_{x} \\
& -1 / 8 \mu m g \operatorname{sgn}(\dot{\theta})(1+1 / 2 \sqrt{2})-m L \dot{\theta}^{2} \\
F_{c y}= & (-3 / 8 \cos (\theta)-5 / 8 \sin (\theta)) m a_{x} \\
& -1 / 8 \mu m g \operatorname{sgn}(\dot{\theta})(1+1 / 2 \sqrt{2})+m L \dot{\theta}^{2}
\end{aligned}
$$

By maximizing the magnetic force and minimizing the size and mass of the module, the maximum acceleration limit can be increased leading to more reliable reconfigurations. Also, a large attractive force can significantly increase variability allowed in reconfiguration. Since the force of magnets scales with volume, according to the square-cube law, the limit of the active torque will decrease faster than the motion inhibiting torque due to friction as the system scales down in size. This limitation can be overcome by changing the bonding method and by decreasing the friction.

\section{External Actuation Analysis}

\section{A. Motion problem/constraints}

In general, external actuation may be defined as any energy pattern imparted on the environment of a system of modules

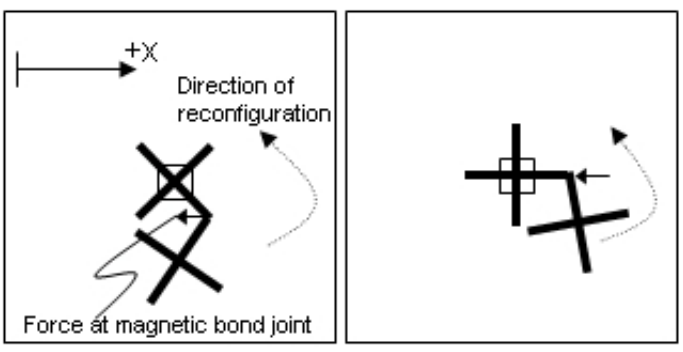

Fig. 6: Two possible orientations for the fixed center module.

that allows them to reconfigure and reach a desired goal configuration in a determined, time bounded manner. The design and construction of a simple one DOF oscillating test apparatus is presented here and both validates the concept, as well as allows the performance of experiments to gather intuition about the hardware and software design issues.

Analysis of the dynamics involved in reconfiguration dictates rough minimum specifications for the maximum acceleration, velocity and travel distance of the linear stage. From this analysis, the requirements for the linear stage is set at $5 \mathrm{~m} / \mathrm{s}^{2}$ minimum acceleration, $2 \mathrm{~m} / \mathrm{s}$ minimum velocity and $0.4 \mathrm{~m}$ minimum travel. In order to minimize the torque necessary to reach a maximum linear acceleration, the whole assembly (table and support) mounted to the guide block must be of minimal mass. A Teflon mat provides a low friction surface attached atop a foam core table which is stiff and light weight. The belt drive, powered by a $50 \mathrm{~W}$ flat EC45 brushless motor with a 26:1 gear reduction from Maxon Motors Inc., meets the requirements.

Given the above specifications for the linear stage table, what is the appropriate a motion pattern or set of motion patterns for each type of reconfiguration? In order for a module to reconfigure, it is necessary to generate a sufficient torque profile (at the magnetic joint connecting the modules) that will cause the module to rotate about one edge of the other module without violating the above constraints. For example, Figure 4 shows a module reconfiguring from the lower right side to the upper right side of the fixed center module. Figure 5 depicts an acceleration profile that causes such a reconfiguration. The table accelerates in the - $x$ direction initially to rotate the module past the halfway $\left(\Delta \theta>90^{\circ}\right)$ point as shown in Figure 4 frame (b). Then, an acceleration in the $+x$ direction causes the module to complete the reconfiguration rotation.

Because the linear stage has one DOF the orientation of the center fixed module is critical as it specifies the distance and direction the magnetic bond joint force acts throughout the reconfiguration. The two symmetric orientation options are shown in Figure 6. The second type of orientation was chosen because it provides the maximum amount of torque at the beginning of the reconfiguration.

A motion profile is composed by developing a table of desired velocities that are fed to the motor control chip at a given frequency. The velocity profiles are developed empirically. For each type of reconfiguration (there are eight: one for each rotation in either direction.) The motion profile may consist of one or two different motion patterns. The goal is to find that profile which is most reliable and falls within 


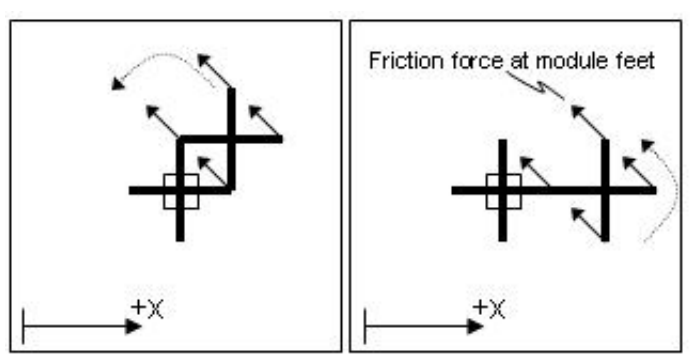

Fig. 7: Two possible singularity conditions.

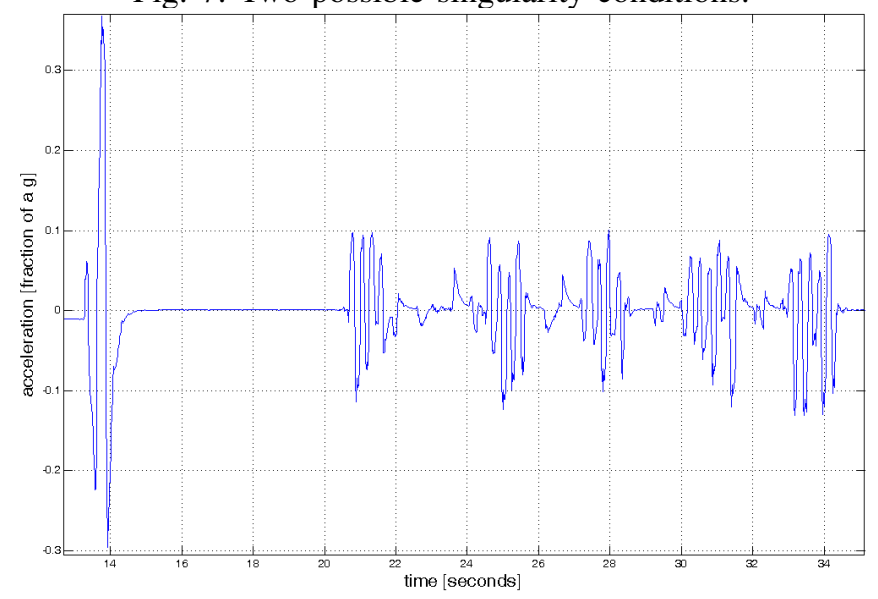

Fig. 8: Acceleration profile for one reconfiguration.

the constraint maximums.

\section{Control Robustness}

\section{A. Issues}

There are several issues that deal with the question of how precise and repeatable the motion pattern has to be in order to ensure that the module can perform any arbitrary reconfiguration. A robust system design should allow for some degree of variability in the motion pattern and still perform a successful reconfiguration.

Because the external actuation system has only one DOF, it is possible for a module to be stopped in a singular condition - the line formed by the center of mass of the module and the rotation point is parallel with the actuated DOF (see Figure 7). There are two types. One type (frame 1, Figure 7) occurs when a module needs to reconfigure to a position that is in the direction of the table's DOF. Experiments have shown that a high magnitude impulses can perturb the module out the first type of singular state. The other case (frame 2, Figure 7) occurs when a module is moving in the direction perpendicular to the table's DOF and gets stuck part way. In this case impulses cause small motions at best and these motions oscillate about the singular point.

\section{EXPERIMENTS}

\section{A. Self-Reconfiguration}

Experiments demonstrated the feasibility of the concept of external actuation and gathered data to validate and improve the design of the system. In all experiments, hand-tuned motion patterns and module algorithms were developed for a specific experiment.
The first experiment demonstrates that external actuation can indeed be used to provide energy for modular robots to self-reconfigure (see Figure 10.) In the experiment, one module is fixed to the center of the linear stage table and another is placed at one of the four possible initial conditions. The goal of the experiment is to have the unfixed module 'walk' clockwise around the fixed module; that is, reconfigure four times by swinging about each leg of the center module successively.

The algorithm to perform this experiment is straightforward and rather robust. The center module acts as a slave and simply serves to help the master module localize himself (as previously explained). The master module continuously checks to see which side is attached to the slave. He then chooses one bond to break with the slave so it rotates about the other bond to a new configuration. This routine is repeated four times. The algorithm is robust in that if a rotation does not move the system to the next state, it will simply start the localization process over and break the appropriate bond to attempt to continue.

Because of the limitations of the module's bonding force and the maximum acceleration of the one DOF stage, several motions are required to cause a module to make a single reconfiguration. Figure 8 shows the motion patterns for the first reconfiguration in a sequence of four reconfigurations. The first part consists of relatively high acceleration burst that swings the module $3 / 4$ of the full reconfiguration rotation. The next part of the motion pattern consists of several steady quick, lower amplitude accelerations that slowly slides the module to its new state.

\section{B. Acceleration profile variability}

In order to make quantitative observations about the degree of variability that the system can handle, 50 reconfigurations were performed. For this experiment, two new modules were used that have significantly stronger magnetic bonds. It was found through successive design iterations that it is possible to design a module (one with stronger magnets and smaller inertia) that is more robust to variations in the motion profile.

The experimental setup consists of two modules, one fixed to the table and one attached at the upper right hand corner of the fixed one. The goal is to generate a motion profile that causes the module to reconfigure in the direction

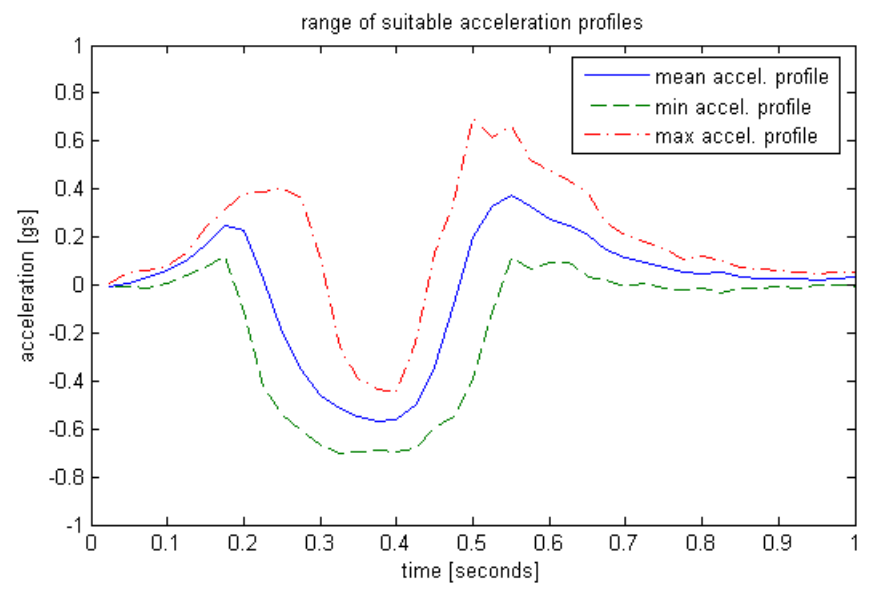

Fig. 9: Mean, max and min acceleration for stronger magnets. 


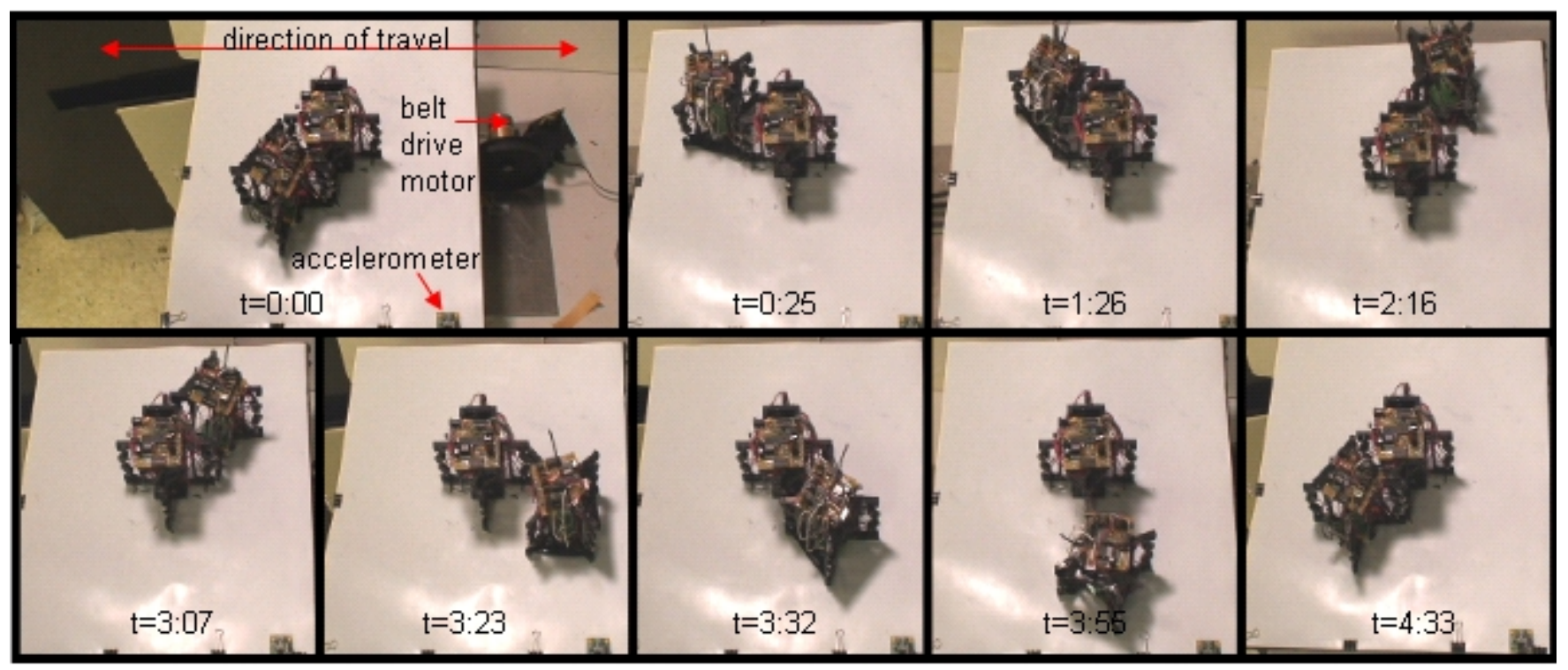

Fig. 10: Sequence of four reconfigurations. The experimental setup can be seen in the first frame. One module begins attached to the center, table-fixed module and reconfigures 4 times caused by the acceleration of linear stage table.

perpendicular to the direction of the linear stages DOF. This motion profile is repeated and the acceleration profile of the table is recorded for each reconfiguration. Due to the natural variability of the system related to the motor, the open loop controller, etc. each run varies significantly enough such that occasionally the motion will not be sufficient enough to cause reconfiguration. The graph in Figure 9 shows the mean, maximum and minimum acceleration profile for the 50 successful reconfiguration runs. The acceleration data consists of 120 samples taken over the 1 second duration of the reconfiguration. A linear interpolation is used to break the time frame into 40 discrete samples and the statistics are taken on these discrete samples. The graph shows that even with the natural variability of the acceleration profile, the MSR robotic system can still successfully reconfigure.

\section{CONClusions And Future Work}

The development of MSR robotic systems that consist of a large number of small scale modules is a prominent goal in the field. The use of external actuation to provide the energy for a MSR system provide a reasonable way to greatly reduce the size requirements (actuation and onboard power) without sacrificing realtime performance. Experiments with a proof of concept system have shown that an appropriate energy profile can be developed that causes sufficient forces to allow a module to reconfigure. In addition, the design process and experimental observations highlight critical design parameters for an externally actuated module.

There are several directions for future work. Experiments will be run to further characterize the balance between critical module parameters (e.g. bonding force, inertia, friction) and the system energy. Near term work includes construction of a 2DOF actuated motion platform to find 2D acceleration profiles. Higher level motion planning algorithms for more complex reconfiguration will be developed in addition to a dynamic simulator in order to study the issues associated with a system of a large number of modules. More modules will be constructed in order to explore software design issues such as the communication scheme and the implementation of reconfiguration algorithms. The development of 3D systems and systems at progressively smaller scales is also in the scope of future work.

\section{ACKNOWLEDGMENT}

The authors wish to thank Intel Research Pittsburgh for sponsoring this project in part.

\section{REFERENCES}

[1] M. Yim, D. Duff, and K. Roufas, "Polybot: a modular reconfigurable robot," in Proc. of the IEEE Intl. Conf. on Robotics and Automation, San Francisco, Apr. 2000, pp. 514-520

[2] M. Yim, Y. Zhang, J. Lamping and E. Mao, "Distributed Control for 3D Metamorphosis," Autonomous Robots, vol 10, no 1 pp. 41-56, Jan. 2001

[3] D. Rus and M. Vona, "Crystalline Robots: Self-Reconfiguration with Compressible Unit Modules," Autonomous Robots, vol 10, no 1 pp. 107-124, Jan. 2001

[4] C. Unsal and P.K. Khosla, "Mechatronic design of a modular selfreconfiguring robotic system," in Proc. of IEEE Intl. Conf. on Robotics and Automation (ICRA) pp. 1742-1747, San Francisco, CA April, 2000

[5] P. Pillai and J. Campbell, "Sensing and reproducing the shapes of 3D objects using claytronics," in Proc. of the 4th Intl. Conf. on Embedded networked sensor systems. pp. 369 - 370, 2006

[6] S.C. Goldstein, J.D. Campbell, and T.C. Mowry "Programmable Matter," IEEE Computer, vol. 28, no. 6, pp. 99-101, May 2005

[7] J. Bishop, S. Burden, E. Klavins, R. Kreisberg, W. Malone, N. Napp, and T. Nguyen, "Self-organizing programmable parts," in Proc. of IEEE/RSJ Intl. Conf. on Intelligent Robots and Systems, (IROS)., Maui, Hi, Nov. 2001

[8] P White, V Zykov, J Bongard and H Lipson, "Three dimensional stochastic reconfiguration of modular robots," in Proc. of Robotics Science and Systems. Cambridge, MA, 2005

[9] J.W. Suh, S.B. Homans and M. Yim, "Telecubes: mechanical design of a module for self-reconfigurable robotics," in Proc. of IEEE Intl. Conf. on Robotics and Automation (ICRA) pp. 4095- 4101, April, 2002

[10] S. Murata, E. Yoshida, A. Kamimura, H. Kurokawa, K. Tomita and S. Kokaji, "M-TRAN: Self-reconfigurable modular robotic system," IEEE/ASME Trans. Mech. vol. 7, no. 4, pp. 431-441. 2002.

[11] W-M. Shen, P.Will, "Docking in self-reconfigurable robots," in Proc. of IEEE/RSJ Intl. Conf. on Intelligent Robots and Systems, (IROS). pp. 1049-1054, Maui, Hi, Nov. 2001 